\title{
SPOLM2O19
}

XIX SIMPÓSIO DE PESQUISA OPERACIONAL ELOGÍSTICA DA MARINHA

\section{OTIMIZAÇÃO DA PARADA DE TUES PARA MANUTENÇÃO CORRETIVA}

\author{
Fábio de Rezende Francisco \\ IME - Instituto Militar de Engenharia \\ Praça Gen. Tibúrcio, 80 - Urca, Rio de Janeiro - RJ \\ fabiorfrancisco1@gmail.com
}

\section{Gustavo Galvão Vasconcelos}

Unigranrio

Rua Prof. José de Souza Herdy, 1.160 - Duque de Caxias, Rio de Janeiro - RJ gugagalvao@msn.com

\author{
Orivalde Soares da Silva Júnior \\ IME - Instituto Militar de Engenharia \\ Praça Gen. Tibúrcio, 80 - Urca, Rio de Janeiro - RJ \\ orivalde@ime.eb.br
}

\begin{abstract}
RESUMO
Dentre as várias dificuldades encontradas no planejamento de parada de (Trem Unidade Elétrica) para manutenção, uma delas é a priorização destes trens considerando diversas restrições como o atendimento à operação, a capacidade de vias em oficina de manobras. Considerando este cenário, o artigo propõe a criação de um modelo matemático utilizando os conceitos de programação linear para otimização de parada de trens para manutenção corretiva, levando-se em consideração as seguintes restrições: trens com avaria crítica; trens com dois ou mais carros com sistema de freio isolado; capacidade de linhas em oficina; além de pontuação total maior que o percentual de referência determinado, para o caso de avarias não críticas. O sistema metroviário do Rio de Janeiro possui, em sua base de dados de manutenção, o registro de ordens de serviço com suas devidas prioridades, que considera o nível de impacto de cada tipo de falha. Para definição de pontuação dos trens a serem encaminhados à oficina, foram atribuídos pontos a partir das prioridades das ordens de serviço, garantindo a parada de trens mais críticos ao respeitar as restrições elencadas através do modelo matemático desenvolvido. O modelo proposto mostrou-se eficaz, tendo em vista que selecionou os trens prioritários, otimizando a parada de trens, considerando as restrições definidas. A utilização deste modelo na rotina diária de programação de TUEs trará benefícios para o processo decisório de parada de trens de um sistema metroferroviário, ao estruturar qualquer processo que se baseie na experiência técnica do programador, que tende a ser limitada, pois não é capaz de considerar todas as restrições envolvidas.
\end{abstract}

Palavra-chave: otimização; manutenção; operação; trens. 


\begin{abstract}
Among several difficulties founded in the planning of TUEs (Electrical Unit Train) maintenance stop, one of them is the prioritization of these trains considering several restrictions such as service attendance, workshop track capacity, maneuvers and so on. Considering this scenario, this article proposes the creation of a mathematical model, using linear programming concepts for train stop optimization for corrective maintenance, taking into account the following restrictions: trains with critical faults; trains with two or more cars with an isolated brake system; workshop line capacity; and total score higher than the percentage reference determined in case of non-critical failures.

The subway system in Rio de Janeiro has, in its maintenance database, the record of service orders with their proper priorities, which considers the level of impact of each type of failure. For the definition of the ranking of the trains to be forwarded to the workshop, scores were assigned based on the priorities of the service orders, ensuring the stop of the most critical trains, while respecting the elected restrictions through the developed mathematical model. The proposed model proved to be effective, take into account that it selected the most critical trains, optimizing the train stop, considering the defined restrictions. The use of this model in the daily programming routine of TUEs will bring benefits to the decision-making of trains stopping process of a subway system as it structures any process that is based on the technical experience of the programmer, which tends to be limited as it is not able to consider all the involved restrictions.
\end{abstract}

Keywords: Optimization; Maintenance; Operation; Trains.

\title{
Como Citar:
}

FRANCISCO, Fábio de Rezende; VASCONCELOS, Gustavo Galvão; SILVA JÚNIOR, Orivalde Soares da. Otimização da parada de TUEs para manutenção corretiva. In: SIMPÓSIO DE PESQUISA OPERACIONAL E LOGÍSTICA DA MARINHA, 19., 2019, Rio de Janeiro, RJ. Anais [...]. Rio de Janeiro: Centro de Análises de Sistemas Navais, 2019.

\section{INTRODUÇÃO}

O uso do transporte coletivo contribui para a diminuição de veículos trafegando nas ruas e avenidas, sujeitas a constantes congestionamentos. Dentre os meios de transporte mais utilizados nos grandes centros está o metrô. Segundo Olortegui (2012), “o sistema metroviário apresenta-se desde a segunda metade do século XX como um sistema eficiente, na medida em que atende às necessidades de deslocamento de maiores contingentes, garante os benefícios dos sistemas de transportes tradicionais e atende às expectativas baseadas em novas solicitações dos "clientes de transportes urbanos", com a apresentação de novos atrativos, como assiduidade, segurança, conforto e higiene".

A regularidade do intervalo dos trens é uma das grandes vantagens competitivas dos sistemas metroviário e ferroviário, se comparados ao sistema rodoviário, sendo este mais suscetível à externalidades que aqueles, por estarem normalmente contido em túneis e faixas de domínio segregadas. Esta vantagem competitiva está intimamente ligada à confiabilidade dos seus ativos. De acordo com Valente (2008), os parâmetros mais importantes em relação ao transporte público, sob o ponto de vista do usuário são os seguintes: confiabilidade, tempo, acessibilidade, conforto, conveniência, segurança e custo.

Coca e Torres (2004) propõem um modelo de avaliação de desempenho nos transportes urbanos baseado em 12 fatores que influem na qualidade do transporte público urbano, sendo um destes 
fatores a confiabilidade. Na definição deste parâmetro é considerada a pontualidade e a efetividade na programação horária pré-estabelecida pela operadora (Coca e Torres, 2004).

É fato que, desde que a segurança da operação não seja afetada, o binômio confiabilidade $\mathrm{x}$ custo precisa ser minuciosamente avaliado para que seja investido no ativo valores que não inviabilizem o negócio no qual este ativo está inserido. Este binômio é muito bem trabalhado pela norma NBR ISO 55001. Segundo o UIC Railway Application Guide (Guia de aplicação da norma ISO 55001 para ferrovias), o efetivo gerenciamento dos ativos permite a uma organização tomar decisões corretas, no tempo correto, para atingir os melhores valores para a organização e seus stakeholders, e isto significa obviamente auferir as melhores receitas, com os melhores custos empenhados nestes ativos.

Torna-se então premente a necessidade de se avaliar custos de manutenção nas empresas, inclusive as do setor metroviário, o que leva a entender que, dependendo do impacto e do nível de segurança tolerado, nem todas as falhas serão mitigadas e por este motivo, manutenções corretivas farão parte do negócio, demandando por parte da administração destas empresas um gerenciamento da manutenção que garanta a minimização dos impactos na operação.

Neste contexto, considerando a necessidade de um suporte sistêmico que elimine as imprecisões geradas por controles manuais e a necessidade de formulação de diretrizes que orientem a tomada de decisão após a ocorrência de uma falha, este artigo propõe um modelo de otimização da programação de parada de trens para manutenção corretiva.

O artigo está organizado da seguinte forma. Na seção 2 apresentam-se as características operacionais do sistema estudado, características estas que são comuns à grande parte dos sistemas metroviários existentes. Na seção 3 apresentam-se o fluxo de tratamento de corretivas no Metrô Rio e o método criado para definição da pontuação destas ocorrências. Na seção 4 o modelo matemático proposto é apresentado, o qual foi testado com em dados reais da empresa MetroRio, e apresentado na seção 5. Na seção 6 os resultados deste teste são analisados, e por fim, na seção 7, apresentam-se as considerações finais, as limitações do modelo e sugestões de aplicação.

\section{CARACTERÍSTICAS OPERACIONAIS DO METRÔ DO RIO DE JANEIRO}

Quando foi inaugurado, em março de 1979, o Metrô do Rio de Janeiro contava com 4,3 quilômetros de trilhos ligando cinco pontos próximos da cidade. Nos primeiros dez dias de operação, seus trens transportaram mais de meio milhão de pessoas, com uma média diária de 60 mil clientes. Atualmente, a malha ferroviária carioca tem um total de 53 quilômetros de extensão e 41 estações, transportando cerca de $800 \mathrm{mil}$ pessoas/dia por 3 linhas que se interligam entre si: a Linha 1 que se estende da Estação Uruguai a Estação General Osório, Linha 2 que vai da estação Pavuna até a estação Botafogo, compartilhando estações com a Linha 1 do trecho de Central a Botafogo e a Linha 4 que pode ser considerada uma extensão da Linha 1 indo da Estação General Osório até a Estação Jardim Oceânico. A Figura 1 mostra a distribuição de linha do sistema Metroviário do Rio de Janeiro.

A MetroRio é a empresa que detém a concessão da operação e manutenção da malha metroviária da cidade do Rio de Janeiro desde 1998, com prazo até 2038. Dentre os ativos desta concessão estão os 64 trens da frota, também conhecidos como TUE (Trem Unidade Elétrica) divididos basicamente em 34 trens fabricados pela empresa chinesa CRC (Changchun Railway Vehicles CO. LTD.) a partir do ano 2012 e 30 trens fabricados pelas empresas Alstom e Mafersa nas décadas de 70 e 80. 


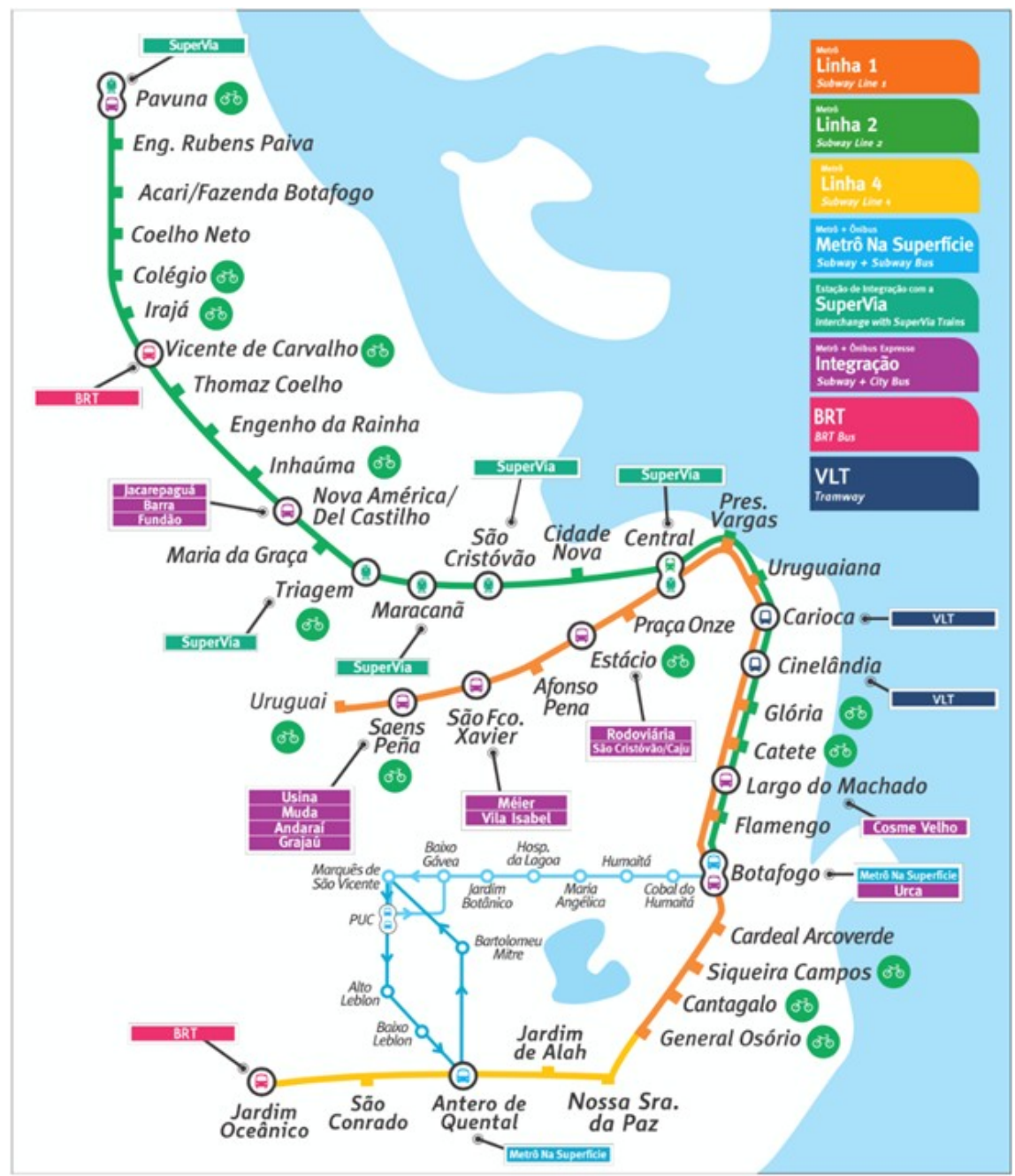

Figura 1: Esquema do sistema metroviário do Rio de Janeiro

Tanto no metrô carioca, quanto na maioria das operações metroviárias, um dia útil é composto de picos e vales de passageiros, sendo o pico manhã o fluxo das zonas habitacionais para os centros comerciais, e o pico tarde, o retorno destes passageiros às zonas de habitação. Entre estes picos estão os vales onde existe uma movimentação de passageiros inferior à movimentação nos picos, o que permite o aumento do intervalo entre composições e a consequente disponibilização de trens para que se cumpram os processos de manutenção.

Três são os tipos possíveis de manutenção de trens e equipamentos em geral: preditiva, preventiva e corretiva. Tanto a manutenção preventiva, quanto à manutenção preditiva acontecem antes que a falha ocorra, sendo que a manutenção preditiva tende a ser mais precisa e por consequência menos custosa, pois atua nas condições de um equipamento de acordo com alterações em parâmetros de controle. Segundo Yan et al. (2008), a manutenção corretiva é realizada após a falha do equipamento. O propósito de manutenção corretiva é trazer o equipamento de volta a um estado funcional assim que possível, reparando ou substituindo o equipamento defeituoso. Este tipo de manutenção é geralmente realizada através de uma intervenção de curto prazo. Ao contrário do que se possa imaginar, não necessariamente as intervenções corretivas são procedimentos de emergência, pois podem também ser realizadas para corrigir e restaurar o desempenho dos equipamentos.

É fato que durante um dia típico de um sistema metroviário, várias são as ocorrências que trazem impacto à operação e prejudicam a entrega da qualidade do serviço ao cliente. 
Alguns dos motivos são: obsolescência de ativos, avaria de sistemas, comportamento de passageiros, impactos externos, etc.

Ao final do pico da manhã da operação comercial do MetroRio, alguns trens precisam ser direcionados para o centro de manutenção para intervenções corretivas, porém, dependendo de como foi a quantidade de avarias no pico manhã, o responsável pelo direcionamento de trens tem a tarefa de atender às demandas corretivas através de manipulação de planilhas eletrônicas geradas pelo sistema de manutenção, considerando diversas restrições tais como: quantidade de vias disponíveis na oficina de manutenção, disponibilidade de mão de obra, tempo previsto para cada tipo de intervenção e o cumprimento do plano de preventivas.

Belhot e Campos (1995) afirmam que a manutenção corretiva não pode ser programada, dada a natureza probabilística da falha e as incertezas que cercam a tomada de decisão correspondente. Termina-se por concluir que é essencial a formulação de diretrizes de manutenção que orientem a tomada de decisões após a ocorrência de uma falha.

\section{DESCRIÇÃO GERAL DO PROBLEMA E FORMULAÇÃO DO MÉTODO DE PONTUAÇÃO.}

\subsection{CRITICIDADE POR TIPO DE OCORRÊNCIA}

O relato de uma falha durante a operação do sistema metroviários carioca segue o fluxo exposto na Figura 2.

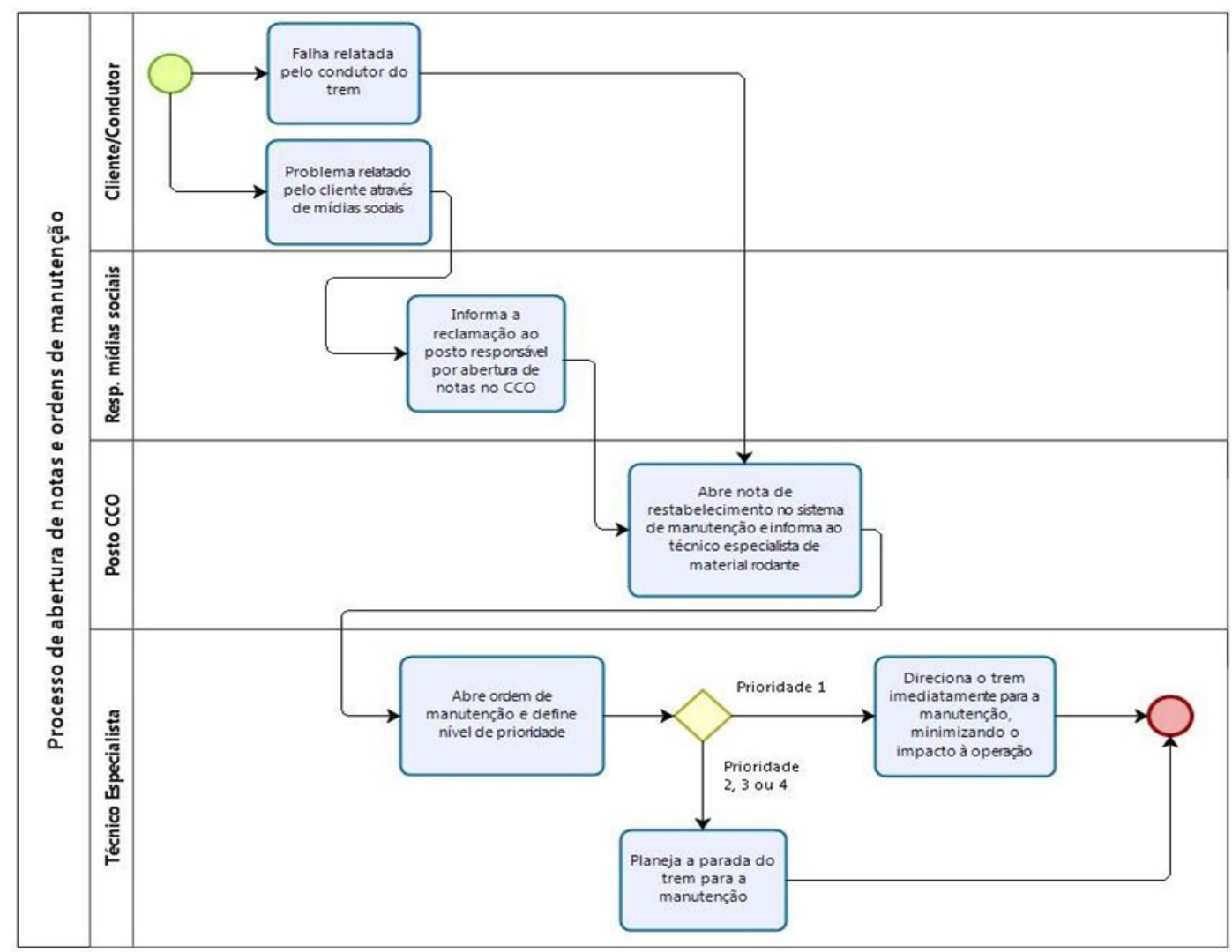

Figura 2: Fluxo de abertura de notas e ordens de manutenção corretiva

As falhas são reportadas tanto pelos condutores dos trens, quanto pelos passageiros através das mídias sociais. Estas falhas são registradas no sistema de manutenção da empresa que no caso é o SAP, através de uma nota de restabelecimento. De posse desta nota, o técnico especialista que dá suporte à operação no centro de controle abre uma ordem de 
serviço e avalia a prioridade de atendimento, que conforme procedimento interno possui a definição relacionada na Tabela 1 .

\begin{tabular}{|c|c|}
\hline Prioridade & Característica da Prioridade \\
\hline P1 - Muito elevado & $\begin{array}{l}\text { Potencial de causar fatalidade ou incapacidade, "Severidade A" (Im- } \\
\text { pacta ou pode impactar na operação comercial. Atraso na operação } \\
\text { comercial superior a } 5 \text { min, evacuação de composição). }\end{array}$ \\
\hline P2 - Elevado & $\begin{array}{l}\text { Potencial de causar lesão séria com incapacidade temporária/perda per- } \\
\text { manente, "Severidade B” (impacta ou pode impactar no fluxo de clien- } \\
\text { tes na estação ou embarque. Lotação de estação/plataforma, Acessibili- } \\
\text { dade); impacta ou pode impactar na operação comercial (atraso na } \\
\text { operação comercial inferior a } 5 \text { min, necessidade de operação manual } \\
\text { de sistema automatizado); Gera desconforto para os clientes (ar con- } \\
\text { dicionado ou ventilação). }\end{array}$ \\
\hline P3 - Médio & $\begin{array}{l}\text { Potencial de causar lesão leve não incapacitante, "Severidade C"; (pro- } \\
\text { voca indisponibilidade de uma redundância. Operação sem redundân- } \\
\text { cia); impacta ou pode impactar no desempenho, mas não causa in- } \\
\text { disponibilidade do sistema; Gera desconforto para os clientes (som, } \\
\text { iluminação, limpeza ou vibração). }\end{array}$ \\
\hline P4 - Baixo & Sem impactos listados nos itens anteriores. \\
\hline
\end{tabular}

Tabela 1: Definição de prioridade de ordens e característica

\subsection{CRITÉRIO DE PONTUAÇÃO}

Utilizando as prioridades da Tabela 1, foram definidas pontuações para cada tipo de prioridade, assim foi realizado em Borges (2013) para o sistema ferroviário de carga. Ressalta-se que o sistema metroviário possui características diferentes do sistema ferroviário de carga, que em caso de avaria de um vagão, o mesmo é substituído no pátio de manobra mais próximo. Já no caso do sistema metroviário, quando uma falha num carro de um trem é constatada, o trem é direcionado para a oficina e fica indisponível até que esta falha seja reparada ou isolada para que o trem volte a operar sem impacto à operação. No caso do MetroRio, como todas as ocorrências já são priorizadas pelos técnicos de manutenção, de acordo com o critério pré-definido na Tabela 1 . Foram atribuídos pontos tão somente para diferenciar os níveis de prioridade entre si, conforme apresentado na Tabela 2.

\begin{tabular}{lr}
\hline \multicolumn{1}{c}{ Prioridade } & Pontuação \\
\hline 1- Muito eleva- & 500 \\
do & \\
2- Elevado & 6 \\
3- Médio & 4 \\
4- Baixo & 2 \\
\hline
\end{tabular}

Tabela 2 - Pontuação por tipo de prioridade e atendimento

\subsection{IDENTIFICAÇÃO DOS TRENS ELEGÍVEIS PARA MANUTENÇÃO}

Borges (2013) define três critérios que sinalizam a necessidade da parada de um trem para realização da manutenção corretiva numa ferrovia:

1) Ocorrência de trem com ordem prioridade 1;

2) Ocorrência de trem com número de carros isolados $\geq$ quantidade de referência.

3) Ocorrência de trem com somatório de P2, P3 e P4 > que a pontuação de referência. 
A pontuação total de um trem é definida pelo somatório da pontuação de cada carro.

Caso um dos carros de um trem esteja com uma ordem de serviço com prioridade 1 , o trem a que este carro faz parte será automaticamente selecionado para parada. Um levantamento realizado na MetroRio mostrou que para o estudo em questão, a média histórica dos últimos 4 anos, considerando a pontuação sugerida na Seção 3.2, foi de aproximadamente 145 pontos. Para garantir que um carro com prioridade 1 será obrigatoriamente selecionado, atribui-se a esta prioridade 500 pontos. O mesmo acontece com um trem com percentual de carros isolados maior que o percentual de referência, isto é, maior ou igual a dois carros isolados. No caso em questão foram considerados os isolamentos do sistema de tração (propulsão isolada e cilindro de freio isolado), por serem os sistemas mais críticos em termos de segurança da operação, e para esta prioridade foram atribuídos 300 pontos para trens com dois ou mais carros isolados. O percentual de isolamentos foi definido a partir das boas práticas utilizadas nos sistemas metrô ao redor do mundo e que garantem a taxa de frenagem mínima necessária para uma operação segura.

\subsection{IDENTIFICAÇÃO DA PROGRAMAÇÃO IDEAL DE INTERVENÇÕES}

A partir das informações extraídas do sistema SAP, uma tabela dinâmica foi criada utilizando o software Excel, sendo um pequeno recorte do seu resumo apresentado na Tabela 3. Com a pontuação total de cada carro, os trens são ordenados de forma decrescente. Os carros que compõem cada trem também são ordenados de forma decrescente de pontuação. Desta maneira é possível visualizar todos os trens prioritários, e em cada trem, os carros que possuem maior pontuação, os carros que possuem ordens com prioridade 1, bem como trens com 2 ou mais carros isolados, considerando todas as ordens de serviço de corretiva abertas. Realizar esta tarefa de forma manual como é feito atualmente, se torna quase impossível, pois o responsável pela seleção de trens o faz sem conseguir visualizar o peso e o total de falhas registradas para cada carro, e como o cenário de falhas pode mudar a qualquer momento, a tarefa do programador de trens fica ainda mais difícil.

Tabela 3: Resumo extraído do SAP a partir das ordens de manutenção registradas.

\begin{tabular}{|c|c|c|c|c|}
\hline Trem & Carro & $\begin{array}{l}\text { Ocorrência de } \\
\text { P1 }\end{array}$ & Carro Isolado & Soma de Pontuação (P2-P4) \\
\hline \multicolumn{5}{|l|}{ MRO- } \\
\hline \multirow[t]{7}{*}{ T003 } & C1008 & 0 & 0 & 44 \\
\hline & C1018 & 0 & 0 & 22 \\
\hline & C1019 & 500 & 0 & 36 \\
\hline & C1053 & 0 & 0 & 18 \\
\hline & C2043 & 500 & 0 & 36 \\
\hline & C2062 & 500 & 0 & 30 \\
\hline & Total & 500 & 0 & 186 \\
\hline \multirow{8}{*}{$\begin{array}{l}\text { MRO- } \\
\text { T004 }\end{array}$} & & & & \\
\hline & C1003 & 0 & 0 & 38 \\
\hline & C1063 & 0 & 0 & 34 \\
\hline & C2014 & 500 & 0 & 26 \\
\hline & C2054 & 0 & 0 & 44 \\
\hline & C2058 & 500 & 0 & 36 \\
\hline & C2081 & 500 & 0 & 34 \\
\hline & Total & 500 & 0 & 212 \\
\hline \multirow{4}{*}{$\begin{array}{l}\text { MRO- } \\
\text { T005 }\end{array}$} & & & & \\
\hline & C1028 & 500 & 0 & 32 \\
\hline & C1066 & 500 & 0 & 58 \\
\hline & C2018 & 0 & 0 & 42 \\
\hline
\end{tabular}




\begin{tabular}{rrrr} 
C2052 & 0 & 0 & 24 \\
C2088 & 500 & 0 & 46 \\
C2108 & 0 & 0 & 26 \\
\hline Total & $\mathbf{5 0 0}$ & $\mathbf{0}$ & $\mathbf{2 2 8}$ \\
\hline
\end{tabular}

As informações apresentadas na Tabela 3 serão utilizadas no modelo de otimização de parada de trens para manutenção corretiva apresentado neste artigo, respeitando as premissas e restrições impostas pelas peculiaridades da operação do MetroRio.

\section{FORMULAÇÃO MATEMÁTICA DO MODELO}

O modelo construído utiliza como base o modelo proposto por Borges (2013) que propôs a otimização da programação de manutenção de trens de carga de uma ferrovia brasileira com base em confiabilidade. Os parâmetros caracterizam as condições técnicas dos trens e carros que deverão ser considerados nas análises da necessidade de se programar a parada ou não de uma determinada composição para manutenção.

A Tabela 4 mostra os parâmetros de trens em relação à existência de carro isolado com falha prioridade 1, percentual de carros isolados maior que o percentual de carro isolado de referência, pontuação total do trem e os parâmetros de pontuação total dos carros, conforme definido na seção 3.2 .

Tabela 4: Parâmetros de trens e carros

\begin{tabular}{|c|c|c|c|}
\hline $\begin{array}{c}\text { Parâmetros de } \\
\text { trens }\end{array}$ & Descrição & $\begin{array}{c}\text { Parâmetros de } \\
\text { carros }\end{array}$ & Descrição \\
\hline$t 1_{i}$ & $\begin{array}{l}\text { Ocorrência de carro com } \\
\text { prioridade } 1\end{array}$ & $c 2_{i j}$ & $\begin{array}{l}\text { Carro } j \text { do trem } i \text { com sistema de } \\
\text { freio isolado }\end{array}$ \\
\hline$t 2_{i}$ & $\begin{array}{l}\text { \% de carro com sistema } \\
\text { de freio isolado }\end{array}$ & $c 3_{i j}$ & $\begin{array}{l}\text { Pontuação total de cada carro } j \text { do } \\
\text { trem } i\end{array}$ \\
\hline$t 3_{i}$ & $\begin{array}{l}\text { Pontuação das } \\
\text { Prioridades P2, P3 e P4 }\end{array}$ & & \\
\hline$t 4_{i}$ & Pontuação total de trem & & \\
\hline
\end{tabular}

Além da seleção de trens, é importante considerar a quantidade de vagas disponíveis em oficina para receber os trens selecionados. Para atender a esta restrição, foram considerados os parâmetros da Tabela 5.

Tabela 5: Parâmetros de capacidade da oficina

\begin{tabular}{ll}
\hline Parâmetro & \multicolumn{1}{c}{ Descrição } \\
$\mathbf{s}$ & \\
\hline Cof & Capacidade da oficina em quantidade de linhas que podem receber um trem completo \\
Locup & Quantidade de linhas já ocupadas \\
\hline
\end{tabular}

Os critérios de decisão são as regras que devem ser obedecidas pelo modelo na programação de parada de um determinado trem. A Tabela 6 mostra os critérios de decisão de parada de um trem, que deve acontecer caso haja algum carro avariado $\left(t 1_{i}=500=>\right.$ Ocorrência de carro com prioridade 1); caso a quantidade de carros isolados seja maior ou igual que a quantidade de referência $\left(t 2_{i} \geq I r=>\right.$ Quantidade de carros isolados de referência); ou caso a pontuação total do trem seja maior que a pontuação limite de referência $\left(t 3_{i}>\operatorname{Pr}=>\right.$ Pontuação limite de referência). Para a pontuação de referência, foi considerada a média dos números da amostragem utilizada, dado que a média, segundo a 
estatística descritiva, é o número que resume todos os demais, mantida certa propriedade.

Tabela 6: Regras de decisão

\begin{tabular}{lll}
\hline $\begin{array}{l}\text { Critéri } \\
\text { o }\end{array}$ & Descrição & Ação \\
\hline 1 & $t 1_{i}>0$ (Ocorrência de carro com prioridade 1) & $\begin{array}{l}\text { Parar o trem para correção da } \\
\text { avaria }\end{array}$ \\
2 & $t 2_{i} \geq \operatorname{Ir}($ Quantidade de carros isolados de & Retirar isolamentos até que $t 2_{i}<\operatorname{Ir}$ \\
& $\begin{array}{l}\text { referência) } \\
t 3_{i}>\operatorname{Pr} \text { (Pontuação limite de referência) }\end{array}$ & Intervir no trem até que $t 3_{i} \leq \operatorname{Pr}$ \\
\hline
\end{tabular}

O modelo matemático que seleciona e prioriza os trens para parada de acordo com os parâmetros e critérios de decisão foram definidos pelas expressões de (1) a (6). As variáveis de decisão $T r_{i}$ indicam os trens $i$ que deverão ser selecionados para manutenção.

$$
\text { Maximizar } \mathrm{z}=\sum_{i=1}^{n} \operatorname{Tr}_{i} t 4_{i}
$$

s.a.

$$
\begin{aligned}
& \sum_{j=1}^{n} c 3_{j} C_{i j} \geq t 3_{i}-\operatorname{Pr}, \forall i \in\{1, \ldots, n\} \\
& \sum_{j=1}^{n} c 2_{j} C_{i j} \geq \operatorname{Ir}, \forall i \in\{1, \ldots, n\} \\
& \sum_{i=1}^{n} \operatorname{Tr}_{i} \dot{i} i \\
& \sum_{i=1}^{n} \operatorname{Tr}_{i} \leq \text { Cof }- \text { Locup } \\
& \operatorname{Tr}_{i} \in\{0,1\}
\end{aligned}
$$

A expressão (1) apresenta a função objetivo que busca maximizar a pontuação total dos trens a serem disponibilizados para manutenção. As restrições (2) garantem que todo trem com pontuação maior que a pontuação de referência seja selecionado. As restrições (3) garantem que todo trem com a quantidade de carros isolados maior que a quantidade de carros isolados de referência seja selecionado. Já as restrições (4) garantem que um trem com carro avariado, e/ou 2 ou mais carros isolados, e/ou pontuação maior que a pontuação de referência, seja selecionado para manutenção. As restrições (5) garantem que a quantidade de trens selecionados não exceda a quantidade de linhas disponíveis na oficina. As restrições (6) garantem que as variáveis de decisão sejam binárias.

\subsection{LIMITAÇÕES DO MODELO}

O modelo em questão não considera a capacidade da oficina em termos de homemhora disponível, o que significa que o homem-hora disponível seria infinito. Outra limitação seria a quantidade de trens que precisam ser direcionados para oficina para manutenção preventiva, atividade que também faz parte da rotina do programador de trens. 


\section{CASO DE ESTUDO: METRORIO}

Determinado o modelo matemático, a modelagem se deu por meio do Solver, que é um suplemento do Excel. Weingarten e Vecchia (2016) afirmam que no atual contexto histórico, existe uma gama de softwares que possibilitam a resolução de modelos matemáticos lineares e não lineares. Dentre eles, destaca-se o suplemento Solver da planilha Excel. Este recurso, além de fácil manuseio e fácil acesso, apresenta ferramentas poderosas, possibilitando a inclusão e centenas de variáveis. Baseado na Tabela 3, foram escolhidos aleatoriamente 10 trens que serviram para aplicação do modelo. Os trens elencados e seus respectivos parâmetros são apresentados na Tabela 7.

Tabela 7: Trens escolhidos aleatoriamente para aplicação no modelo

\begin{tabular}{rrrrrr}
\hline & $\begin{array}{c}\mathbf{t 1} \\
\text { Trem }\end{array}$ & $\begin{array}{c}\mathbf{t} 2 \\
\text { (Prioridade } \\
\text { (Carros isolados } \geq\end{array}$ & $\begin{array}{c}\text { t3 } \\
\text { (Pontuação }\end{array}$ & Pr) & (Pontuação total) \\
\hline 006 & 500 & 0 & 274 & 774 \\
007 & 500 & 300 & 232 & 1032 \\
013 & 500 & 300 & 212 & 1012 \\
022 & 500 & 0 & 186 & 686 \\
027 & 500 & 300 & 170 & 970 \\
042 & 0 & 0 & 154 & 154 \\
043 & 0 & 0 & 152 & 152 \\
049 & 500 & 0 & 0 & 500 \\
056 & 0 & 0 & 0 & 0 \\
061 & 0 & 0 & 68 & 68 \\
\hline
\end{tabular}

Na Tabela observa-se que os trens que possuem pelo menos um carro com ordem prioridade 1 recebem 500 pontos na coluna "t1 (Prioridade 1)". Da mesma forma, trens com 2 ou mais carros isolados recebem 300 pontos na coluna "t2 (Carros isolados $\geq 2$ )". Finalmente, a pontuação das prioridades dois, três e quatro é apontada na coluna "t3 (Pontuação > Pr)”. 


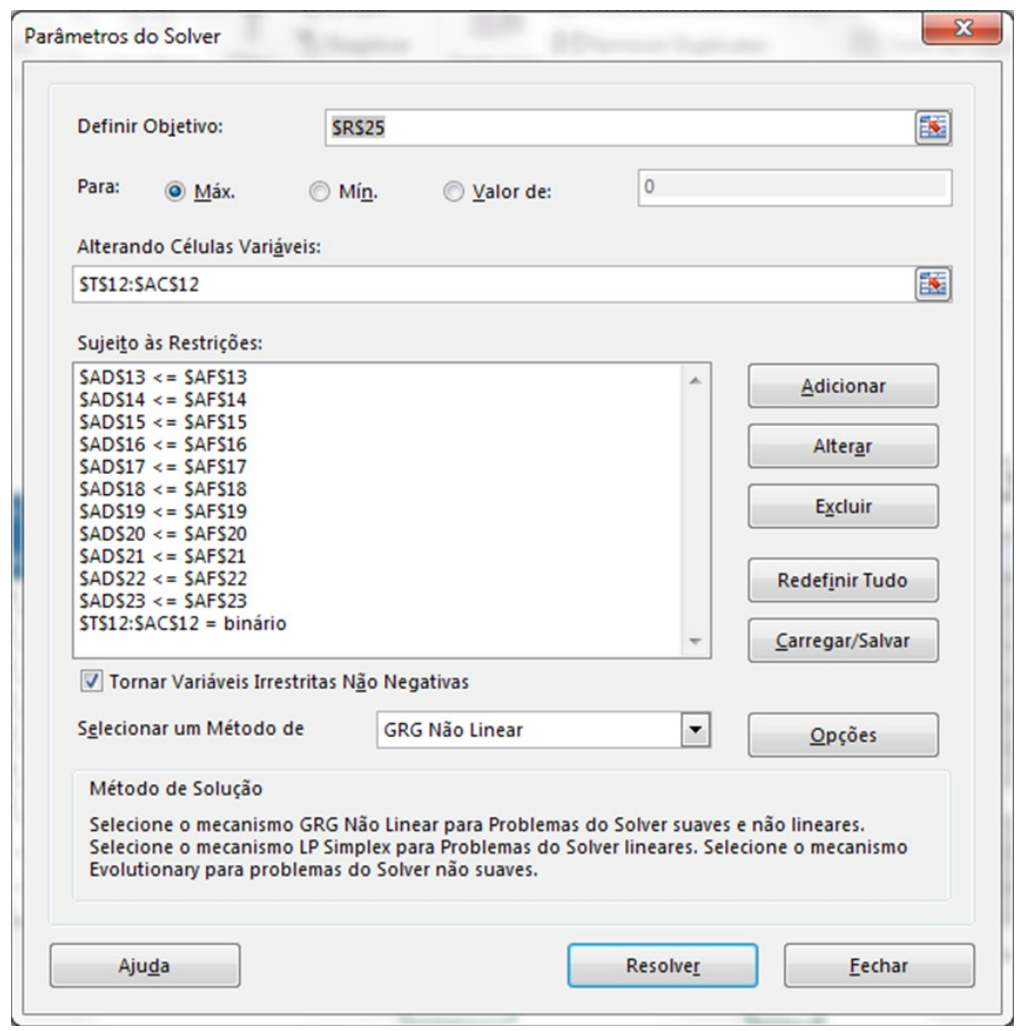

Figura 3: Parâmetros aplicados no software Solver do Excel

O modelo matemático descrito na seção 4 foi construído no software Solver do Excel, utilizando os seguintes valores para os parâmetros Cof $=10$ e Locup $=3$. Os resultados da solução encontrada pelo Solver são apresentados na Tabela 8. A pontuação total foi de 5.128 com um total de 7 trens selecionados.

Tabela 8: Resultado da seleção dos trens obtido com o Solver do Excel

\begin{tabular}{lcccccccccc}
\hline Código dos trens & 00 & 013 & 004 & 012 & 053 & 05 & 029 & 050 & 049 & 062 \\
& 6 & & & & & 5 & & & & \\
\hline Trens selecionados & 1 & 1 & 1 & 1 & 1 & 1 & 1 & 0 & 0 & 0 \\
\hline Pontuação total dos trens & $\mathbf{7 7}$ & $\mathbf{1 . 0 3 2}$ & $\mathbf{1 . 0 1}$ & $\mathbf{6 8 6}$ & $\mathbf{9 7 0}$ & $\mathbf{1 5}$ & $\mathbf{1 5 2}$ & $\mathbf{5 0 0}$ & $\mathbf{0}$ & $\mathbf{6 8}$ \\
& $\mathbf{4}$ & & $\mathbf{2}$ & & & $\mathbf{4}$ & & & & \\
\hline
\end{tabular}

\section{DISCUSSÃO DOS RESULTADOS}

O modelo utilizou como exemplo uma frota com 10 trens, cujas pontuações variaram entre 68 e 1.032 pontos. Na amostragem foram considerados trens pontuados em todos os parâmetros, como também trens pontuados em apenas um parâmetro. Como capacidade da oficina (Cof) considerou-se 10 vagas, sendo que 3 já estariam ocupadas (Locup), desta forma teríamos 7 vagas a serem preenchidas. Utilizando a função objetivo descrita na seção 4, foram selecionados os seguintes trens: 006, 013, 004, 012, 053, 055 e 050, ativos estes que possuíam a maior pontuação, já os trens: 049 e 062 não foram selecionados, pois não possuem pontuação acima das referências citadas. O trem 029, apesar de possuir pontuação acima da pontuação de referência, não foi selecionado por falta de vaga em oficina, já que seria o oitavo trem selecionado. Pelos resultados auferidos, o modelo atende ao objetivo a que se propõe, pois os trens foram selecionados maximizando a pontuação e atendendo às restrições descritas. 


\section{CONSIDERAÇÕES FINAIS}

O objetivo deste artigo foi propor uma solução para otimização de parada de trens para manutenção corretiva utilizando os conceitos de programação linear para chegar ao número ótimo de trens a serem direcionados para a manutenção. Para tanto, foram selecionados dados de ordens de manutenção residentes no sistema de manutenção da empresa MetroRio, gerando com estas ordens uma tabela dinâmica com as informações necessárias ao modelo proposto. A partir daí definiram-se parâmetros e critérios de decisão para seleção e priorização de trens.

Apesar do estudo de caso considerar apenas 10 trens, o número de trens que o modelo pode considerar pode ser bem maior, considerando apenas a capacidade de processamento do software a ser utilizado. Neste trabalho utilizou-se como ferramenta o software Solver do Excel. As limitações deste modelo constituem-se em oportunidade para aprimoramento da solução proposta, de maneira a entregar ao programador de trens uma seleção ainda mais adequada à realidade da manutenção e à necessidade da operação de trens, sem impacto ao passageiro que será sempre o beneficiário final.

Como sugestão, a determinação da pontuação por cada tipo de prioridade pode ser estabelecida a partir das técnicas de Manutenção Centrada em Confiabilidade (MCC) que segundo Seixas (2002) é a aplicação de um método estruturado para se estabelecer a melhor estratégia de manutenção para um dado sistema ou equipamento. O método permite avaliar a criticidade das falhas e onde é possível identificar consequências significantes que afetam a segurança, a disponibilidade ou o custo. Para tanto, será preciso hierarquizar cada sistema a ser analisado, ou seja, identificação do sistema, subsistema, item, componente e peça.

Com o modelo proposto neste artigo, é possível que o responsável pela seleção de trens consiga, com apoio sistemático e metodológico, encaminhar para manutenção os trens que realmente sejam mais prioritários em termos de falhas. A utilização deste modelo na rotina diária de programação de TUEs trará benefícios para o processo decisório de parada de trens de um sistema metro ferroviário ao estruturar qualquer processo que se baseie na experiência técnica do programador, que tende a ser limitada, pois não é capaz de considerar todas as restrições envolvidas.

\section{REFERÊNCIAS BIBLIOGRÁFICAS}

[1] OLORTEGUI, José Antonio Chavez; RODRIGUES, Evaldo Cesar Cavalcante; JÚNIOR, Aldery Silveira; SHIMOISHI, José Matsuo. Política pública de transporte metroviário como fator de impacto no mercado imobiliário e na arrecadação de impostos. [Brasília, DF]: PANAM, 2012, 13 p.

[2] Valente, Matar Amir. Qualidade e produtividade nos transportes. 2. Ed. São Paulo: Cengage Learning, 2015. 239 p.

[3] COCA, Antônio Clóvis; TORRES, Isaac Guilhermino Espinosa. Transporte Público Urbano. 2. ed. São Carlos: Rima, 2004. 428 p.

[4] UIC INTERNATIONAL UNION OF RAILWAYS. UIC Railway application guide Practical implementation of asset management through ISO 55001. Paris, 2016. 96p.

[5] YAN, Shangyao; CHEN, Chun Ying; YUAN, Chun Ying. Long-term aircraft maintenance scheduling for an aircraft maintenance centre: a case study. International Journal of Applied Management Science, v.1, n. 2, 2008, 143 p. 
[6] BELHOT, Renato Vairo; CAMPOS, Fernando Celso de. Relações entre manutenção e engenharia de produção: uma reflexão. Prod. [online]. 1995, v.5, n.2, p.125-134.

[7] BORGES, Gustavo Fernando de Oliveira. Otimização da programação de manutenção de trens com base em confiabilidade. 2013. 93 p. Trabalho apresentado como como requisito parcial para a obtenção do título de Mestre em Ciências em Engenharia de Transportes. Pós-graduação em Engenharia de Transportes, IME, Rio de Janeiro, 2013.

[8] BODANESE, Ronaldo E.; OLIVEIRA, José A.; SCALABRIN, Idionir; MORAES, Claudionor J.. [2005]. Teoria das restrições, pesquisa operacional e programação linear, estudo de caso com a utilização do solver. In: IX Congresso Internacional de Custos, Florianópolis, 13 p., 2005.

[9] WEINGARTEN, Tiago; VECCHIA, Rodrigo Dalla. Modelagem matemática e pesquisa operacional: uma possibilidade. In: Encontro Nacional de Educação Matemática, São Paulo, 12 p., 2016.

[10] SEIXAS, Eduardo de Santana. Manutenção Centrada Na Confiabilidade - Estabelecendo a política de manutenção com base nos mecanismos de falha dos equipamentos. Artigo, RELIASOFT. São Paulo, 10 p. 2010. 